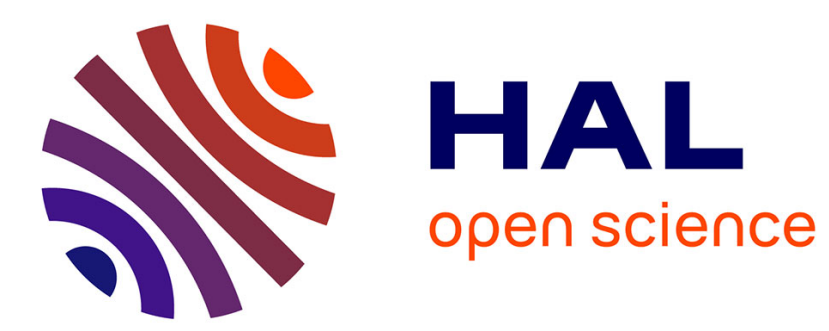

\title{
MARTENSITIC DOMAIN BOUNDARIES IN SHAPE-MEMORY ALLOYS AS SOLITARY WAVES
}

\author{
F. Falk
}

\section{To cite this version:}

F. Falk. MARTENSITIC DOMAIN BOUNDARIES IN SHAPE-MEMORY ALLOYS AS

SOLITARY WAVES. Journal de Physique Colloques, 1982, 43 (C4), pp.C4-203-C4-208. 10.1051/jphyscol:1982425 . jpa-00222139

\section{HAL Id: jpa-00222139 \\ https://hal.science/jpa-00222139}

Submitted on 1 Jan 1982

HAL is a multi-disciplinary open access archive for the deposit and dissemination of scientific research documents, whether they are published or not. The documents may come from teaching and research institutions in France or abroad, or from public or private research centers.
L'archive ouverte pluridisciplinaire HAL, est destinée au dépôt et à la diffusion de documents scientifiques de niveau recherche, publiés ou non, émanant des établissements d'enseignement et de recherche français ou étrangers, des laboratoires publics ou privés. 
JOURNAL DE PHYSTQUE

F. Fa1k

Universität-GH-Paderborm, $F B$ 6, D-479 Paderborn, F.R.G.

(Revised text accepted 23 August 1982)

Abstract.-Based on a one-dimensional model of shape-memory alloys, domain boundaries between martensite variants as well as between austenite and martensite are treated as solitary waves.

Introduction. -The shape memory effect and related phenomena such as pseudoelasticity are due to a first order martensitic phase transition /1/. The high temperature phase (austenite) of most of the alloys (e.g. AuZn, AuCd, NiTi, CuAlZn) showing these effects has an ordered bcc crystal lattice (CsCl or $\mathrm{Fe}_{3} \mathrm{Cl}$ ). The low temperature phase (martensite) shows orthorhomb ic symmetry $(9 \mathrm{R}, 18 \mathrm{R}, 3 \mathrm{R}, 2 \mathrm{H})$. The phase transition is connected with a shear parallel to the habit plane (near (110) in [110]-direction). The martensitic phase transition can be induced not only by cooling but also by applying an external stress which favours one of the martensite variants. According to the first order phase transition we have hysteresis in the temperature as well as in the stress induced transformation. We know from experiments that inside the hysteresis loop the crystal shows a domain structure that is, different martensitic and austenitic domains coexist. Changing temperature or applied stress we alter the equilibrium configuaration of the domains. According?y, some domains grow, by domain boundary movement, at the expense of other ones. In the present paper, with in the framework of a one-dimensional Ginzburg-Landau mode1, domain boundaries are treated as solitary waves.

One-dimensional Ginzburg-Landau theory. Qualitatively the observed phenomena in shape-memory alloys such as pseudoelasticity, ferroelasticity, lattice softening etc. can be described by a one-dimensional model already. We restrict ourselves to one habit plane and to a shear strain in one direction, that is to austenite and two martensite variants $/ 2 \%$. The crystal is built up by stacking of atomic planes which are parallel to the habit plane. For an homogeneously deformed crystal of constant temperature the following Landau free energy density, with shear strain e serving as order parameter, proved to agree with experiments /2/

$$
F_{L}(e, T)=e^{6}-e^{4}+(T+1 / 4) e^{2}
$$

For $F_{L}$, e, and temperature $T$, rescaled units are used. The rescaling parameters depend on the specific alloy $/ 2 / . F_{L}(e, T)$ is plotted, as a function of shear strain $e$ for different temperatures in Fig. 1. For $T>1 / 12 \mathrm{FL}$ has one austenitic (A) minimum at $e=0$ only. For $T<-1 / 4 F_{1}$ has two martensitic minima, corresponding to the right and left martensite twin $\left(M^{+}, M^{-}\right)$. For $-1 / 4<T<1 / 12$ there are three minma, one austenitic and two martensitic ones. At $T=0$ all the minima are of equal depth that is, austenite and martensite are in equilibrium.

If we deal with doma in walls the Landau free energy (1) does not make sense since we would get walls both of vanishing width and of vanishing energy, which is not confirmed oy experiments. This fact shows that Landau free energy is appropriate to situations only where the strain does not vary rapidly. In order to describe domain boundaries we have to add to (1) a term containing the strain gradient $e^{\prime}$ to get a Ginzburg-Landau free energy density

$$
F\left(e, e^{\prime}, T\right)=F_{L}(e, T)+e^{, 2}
$$


From (2) we get the shear stress $\sigma$ and the couple stress $\mu$

$$
\begin{aligned}
& \sigma(e, T)=\partial F / \partial e=6 e^{5}-4 e^{3}+2(T+1 / 4) e \\
& \mu\left(e^{\prime}\right)=2 e^{\prime}
\end{aligned}
$$

Static walls. - If there is no external force and if the strain does not change in time the total free energy of the crystal is given by

$$
F_{\text {tot }}=\int_{x_{1}}^{x_{2}} F\left(e(x), e^{\prime}(x), T\right) d x
$$

where $x$ denotes the stacking direction in rescaled units. $x_{1}$ and $x_{2}$ are the boundaries of the crystal. The structure $e(x)$ of the domain walls is given by the minimum of $\mathrm{F}_{\text {tot }}$. The corresponding Euler-Lagrange equation reads

$$
\sigma-\mu^{\prime}=0
$$

with boundary conditions

$$
\mu\left(x_{1}\right)=\mu\left(x_{2}\right)=0
$$

The prime denotes derivatives with respect to $x$. Eq. (4) is a nonlinear differential equation for the structure $e(x)$ of the static domain walls which can be integrated to

$$
e^{\prime}=\sqrt{F_{L}(e)-F_{0}}
$$

with a constant of integration

$$
F_{0}=F\left(e\left(x_{1}\right)\right)=F\left(e\left(x_{2}\right)\right) \text {. }
$$

From (5) we get for the inverse function of $e(x)$ the elliptic integral

$$
x(e)=\int \frac{d e}{\sqrt{e^{6}-e^{4}+\left(T+\frac{1}{4}\right) e^{2}-F_{0}}}
$$

Eq. (6) yields, depending on temperature and boundary conditions different types of solutions. In the finite crystal we have two types of periodon solutions that is a periodic arrangement of domain walls, either $M^{+}-M^{-}$or $A-M$.

For $M^{+-} M^{-}$wa11s $(T<0$, Fig. 2) we get

$$
e(x)=\frac{e_{1} e_{2} \operatorname{sn}\left(\beta x, k_{1}\right)}{\left(e_{2}^{2} c n^{2}\left(\beta x, k_{1}\right)-e_{1}^{2}\right)^{1 / 2}}
$$

with sn, cn the Jacobian elliptic functions and

$$
\beta=e_{3} \sqrt{e_{2}^{2}-e_{1}^{2}} \quad k_{1}=e_{2} \sqrt{e_{3}^{2}-e_{1}^{2}} / \beta
$$

$e_{1}$ are the solutions $e_{1}^{2}<0,0<e_{2}<e_{3}$ of

$$
e^{6}-e^{4}+(T+1 / 4) e^{2}-F_{0}=0
$$

The boundary condition yields, with the length of the crystal $L=x_{2}-x_{1}$, and $K$ the complete elliptic integral of the first kind

$$
L=2 n K\left(k_{1}\right) / B
$$

For a given length we get a spectrum of periodon solutions enumerated by $n$ which is the number of domain walls within the crystal. An example of this sort of periodic arrangement of $M^{+}-M^{-}$twins is plotted in Fig. 2.

In the case of $A-M-$ wa $115(T>-1 / 4, F i g .3)$ we have 
with

$$
e(x)=\frac{e_{1} e_{2}}{\left(e_{2}^{2}-\left(e_{2}^{2}-e_{1}^{2}\right) s n^{2}\left(\gamma x, k_{2}\right)\right)^{1 / 2}}
$$

$$
r=e_{2} \sqrt{e_{3}^{2}-e_{1}^{2}} \quad k_{2}=e_{3} \sqrt{e_{2}^{2}-e_{1}^{2} / r}
$$

$e_{i}$ are the solutions $0<e_{1}<e_{2}<e_{3}$ of (7). The boundary condition now yields

$$
L=n K\left(k_{2}\right) / \gamma
$$

Aga in we get a spectrum of periodon solution (Fig. 3). $n$ is the number of A-M-walls within the crystal.

For an infinite crystal we have from (6) four types of static solutions namely (Fig. 4)

1. $M^{+}-M^{-}$kink $(T<0)$

$$
\begin{aligned}
& e^{2}(x)=\frac{e_{0}^{2}\left(2 e_{0}^{2}-1\right) \sinh ^{2} \delta x}{e_{0}^{2}+\left(2 e_{0}^{2}-1\right) \cosh ^{2} \delta x} \\
& \delta=e_{0} \sqrt{3 e_{0}^{2}-1} \quad e_{0}^{2}=1 / 3+\sqrt{1-12 T / 6}
\end{aligned}
$$

2. A-M kink $(T=0)$

$$
e^{2}(x)=1 /\left(2\left(1+e^{-x}\right)\right)
$$

3. Soliton on austenite $(T<0)$

$$
\begin{aligned}
& e^{2}(x)=\frac{e_{2}^{2} e_{3}^{2}}{e_{3}^{2}-\left(e_{3}^{2}-e_{2}^{2}\right) \sinh ^{2} \alpha x} \\
& \alpha=\sqrt{T+1 / 4} \quad e_{2 / 3}^{2}=1 / 2 \pm V-T
\end{aligned}
$$

4. Soliton on martensite $(0<T<1 / 12)$

$$
e^{2}(x)=\frac{e_{0}^{2}\left(1-2 e_{0}^{2}\right)}{e_{0}^{2}-\beta^{2} \tanh ^{2} \delta x}
$$

The total energy of all the types of static domain walls is published eisewhere.

Moving walls. - If the walls are moving we cannot expect temperature to stay constant because of the heat of transformation. Instead we must deal with the adiabatic case where entropy does not vary. However, qualitatively in both cases we get the same results. For convenience we shall assume that temperature is fixed. Then we must extend the Ginzburg-Landau free density (2) by kinetic energy to the Lagrangian

$$
L=\dot{u}^{2} / 2-F\left(e, e^{1}, T\right)
$$

where $u$ is the displacement which is connected with the strain e by

$$
u^{\prime}=\mathrm{e} \text {. }
$$

The dot denotes time derivative. By means of Hamilton's principle we get from (12) the equations of motion

$$
\ddot{u}+\left(\sigma-\mu^{\prime}\right)^{\prime}=0
$$


with boundary conditions

$$
\begin{aligned}
\sigma-\mu^{\prime} & =0 \\
\mu & =0
\end{aligned}
$$

Eq. (13) is a nonlinear evolution equation, which, for an infinite crystals, has solitary wave solutions, that is moving strain waves with stationary shape

$$
e(x, t)=e(x-v t)=e(z)
$$

with fixed velocity $v$. Inserting this ansatz into (13) we get

$$
v^{2} e^{\prime \prime}=\left(\sigma-\mu^{\prime}\right)^{\prime \prime}
$$

where the prime denotes the derivative with respect to the argument $z$. Tedious algebra yields the same four types of solutions as above (Fig. 4).

1. $M^{+}-M^{-}$kink.-Possible are arbitrary velocities if $T<1 / 12$, and $v^{2}>2 T-1 / 6$ if $T>1 / 12$. The structure is the same as in $(8)$, we only have to replace $T$ by $T-v^{2} / 2$.

\section{A-M kink.- Possible for $0<T<1 / 75$ with}

$$
v^{2}=4 \sqrt{T / 3}-8 T
$$

3. Soliton on austenite.- The velocity of the soliton is determined by its ampli-

$$
v^{2}=2 T+\left(1-2 e_{m}^{2}\right)^{2} / 2 .
$$

$e_{m}$ is 1 imited to the range

$$
\begin{array}{ll}
e_{m}^{2}<1 / 2-\sqrt{-T} & -1 / 4<T<0 \\
e_{m}^{2}<1 / 2 & T>0
\end{array}
$$

The structure is given by $(10)$ if we replace $T$ by $T-v^{2} / 2$.

4. Soliton on martensite. $-(T<1 / 12)$. The velocity of the soliton is determined by the strain $e_{m}$ of its maximum

$$
v^{2}=2\left[e_{m}^{4}+2 e_{0} e_{m}^{3}+\left(3 e_{0}^{2}-1\right) e_{m}^{2}+2 e_{0}\left(2 e_{0}^{2}-1\right) e_{m}+e_{0}^{2}\left(2 e_{0}^{2}-1\right)\right]
$$

with $e_{0}$ from (8). $e_{m}$ is limited to the range

$$
\sqrt{1-2 e_{0}^{2}}<e_{m}<e_{0} \quad 0<T<1 / 2 \quad, \quad-e_{0}<e_{m}<e_{0} \quad T<0 .
$$

Discussion. The present dynamic one-dimensional Ginzburg-Landau model is a continuum analog to a discrete model of Suzuki $/ 4 /$. He numerically treated the problem of a crystal containing discrete atomic planes with periodic boundary conditions under the same Lagrangian (12). $\phi^{4}$ and $\phi^{6}$ models, nowadays very common in solid state theory $/ 5 /$, are quite different inspite of the similarity of Lagrangian (12). In those models, the free energy contains the displacement $u$, whereas in this model $F$ depends on the strain $e$, that is on the derivative of $u$.

\section{References}

1 Delaey L., R.V. Krishnan, H. Tas, H. Warlimont, J. Mat. Sci. 9 (1974), 1521

2 Falk F., Acta met. 28 (1980), 1773

3 Falk F., to be published in Arch. Mech. (1983)

4 Suzuki T., J. Phys. Soc. Jpn. 45 (1978), 860

5 Krumhansi J.A., J. R. Schrieffer, Phys. Rev. B11 (1975), 3535 

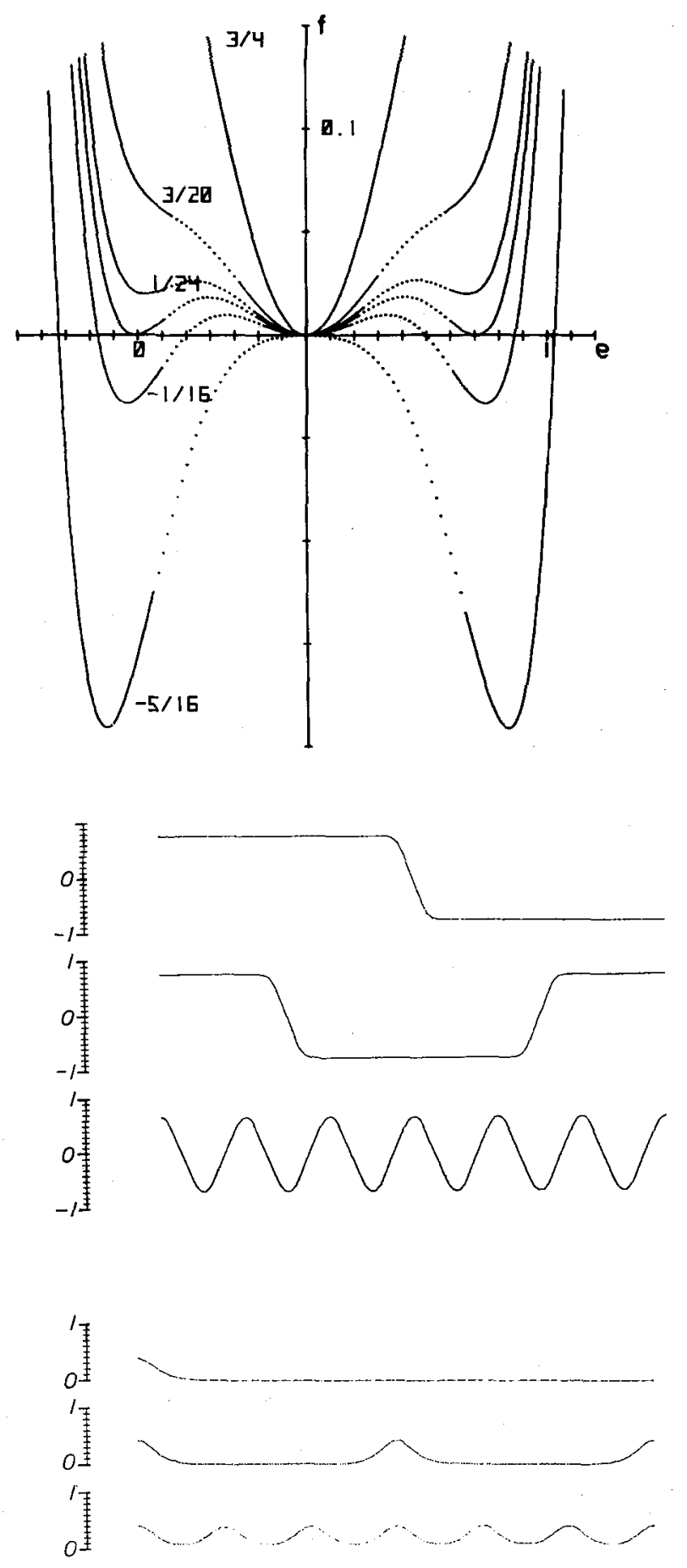

Fig. 1

Landau free energy $F_{L}$ as a function of strain e for different temperatures $T$.

Fig. 2

Structure of $M^{+}-M^{-}$domain walls for temperature $T=-0.1$ and lengtr: of the crystal $L=100$. The strain is plotted over the coordinate $x$ for $n=1,2$, and 12 .

Fig. 3

Structure of A-M domain walls for temperature $T=-0.1$ and length of the crystal $L=100$. The strain is plotted over the coordinate $x$ for $n=1,4$, and 12 . 


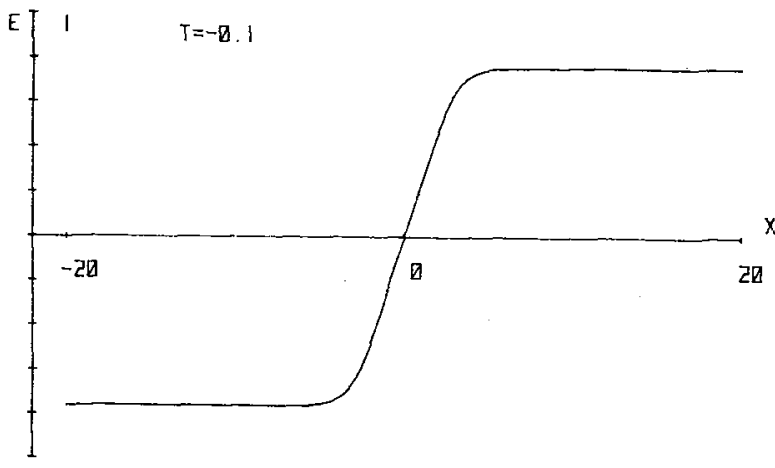

Fig. 4.1

Martensite-martensite kink in an infinite crystal (Eq. 8)

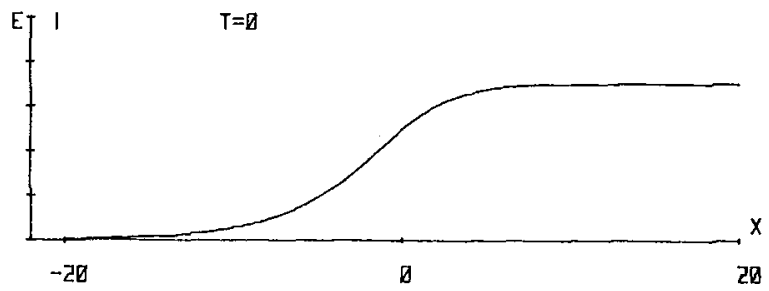

Fig. 4.2

Austenite-martensite kink in an infinite crystal (Eq. 9).

Fig. 4.3

SoTiton on austenite (Eq. 10).

Fig. 4.4

Soliton on martensite (Eq. 11). 